\title{
Efeitos da técnica isostretching em pacientes com escoliose: uma revisão sistemótica
}

\author{
Isostretching technique effects in patients with scoliosis: \\ systematic review
}

\author{
Hitallo Lima da Silva; Flóvia Helena Germano Bezerra; Patrícia Xavier Lima Gomes; Francisco \\ Fleury Uchoa Santos-Júnior \\ Curso de Fisioterapia, Centro Universitário Estácio do Ceará. Fortaleza, CE - Brasil. \\ Endereço para Correspondência: \\ Hitallo Lima da Silva, Rua Eliseu Uchoa Becco - 600, Água Fria \\ 60810-270 - Fortaleza - CE [Brasil] \\ hitalols@hotmail.com
}

\begin{abstract}
Resumo
Introdução: $\mathrm{O}$ isostreching é uma modalidade terapêutica onde o tratamento é focado na melhora dos desequilíbrios posturais. Objetivo: Identificar os efeitos da técnica isostreching no tratamento da escoliose. Métodos: Trata-se de uma revisão sistemática de estudos publicados entre os anos de 1997 e 2017 . Foram utilizados os termos de busca: escoliose, isostretching, isolados e associados em português e em inglês nas bases de dados Google acadêmico, SciELO; PEDro; PubMed; Bireme e Cochrane. Resultados: Durante a pesquisa foram encontrados 314 artigos, após a leitura e os critérios estabelecidos, foram selecionados para compor a revisão 6 estudos. A técnica apresentou resultados positivos em relação ao padrão postural, gibosidade e ângulo de Cobb. Conclusão: Pode-se concluir que a técnica de isostretching na escoliose teve resultados positivos, entretanto os resultados ainda são bastante discutíveis, haja visto que a quantidade de ensaios clínicos que abordaram o tema é baixa.
\end{abstract}

Descritores: Curvaturas da Coluna Vertebral; Postura; Fisioterapia.

\begin{abstract}
Introduction: Isostreching is a therapeutic modality where treatment is focused on the improvement of postural imbalances. Objective: To identify the effects of the isostreching technique in the treatment of scoliosis. Methods: This is a systematic review of studies published between 1997 and 2017. The search terms were: scoliosis, isostretching, isolated and associated in Portuguese and English in the databases Google academic, SciELO; Pedro; PubMed; Bireme and Cochrane. Results: During the research, 314 articles were found, after reading and the established criteria, 6 studies were selected to compose the review. The technique presented positive results in relation to the postural pattern, gibosity and Cobb angle. Conclusion: In this way, the technique of isostretching in scoliosis had positive results, however the results are still quite controversial, since the number of clinical trials that approached the subject is very low.
\end{abstract}

Keywords: Spinal Curvatures; Posture; Physical Therapy Specialty. 


\section{Introdução}

O termo escoliose refere-se as curvaturas laterais da coluna vertebral. Essas curvas ocorrem associadas a torção da coluna vertebral, podendo ser classificada como estrutural, funcional ou idiopática, sendo boa parte de origem desconhecida ${ }^{1}$. A escoliose é mais prevalente em adolescentes com idade 11-18 anos do sexo feminino ${ }^{2}$. Pode ser diagnosticada através de exames radiográficos, através da medida do ângulo de Cobb, com valores de deslocamento superiores a $10^{\circ}$ de desvio lateral ${ }^{3}$.

Os objetivos buscados durante a reabilitação na escoliose, são manter a função e impedir os sintomas a curto e a longo prazo, visando evitar a progressão da deformidade da coluna vertebral ${ }^{4}$. Os tratamentos mais utilizados atualmente são: Reeducação Postural Global $\left(\mathrm{RPG}^{\circledR}\right)^{5}$, Pilates $^{6}$, isostretching ${ }^{7}$, manipulações osteopáticas $^{7}$ e o método Klapp ${ }^{8}$.

O isostretching é uma das modalidades terapêuticas indicadas para tratamento deste tipo de desequilíbrio postural ${ }^{9}$. O método foi criado pelo fisioterapeuta Bernard Redondo em 1974 na França e tem como principal objetivo fortalecer e aumentar a flexibilidade dos grupos musculares. A técnica é realizada com contrações isométricas ou estiramentos de vários músculos associados a uma expiração profunda e um auto alongamento do tronco ${ }^{10}$.

Trata-se de um método postural pois os exercícios são realizados respeitando as curvas fisiológicas da coluna vertebral. Os alongamentos e contrações são incluídos em cada postura trabalhando globalmente o corpo em cada exercício $^{11,12}$. Dessa maneira o método busca trazer um aperfeiçoamento da consciência corporal, controle neuromucular ${ }^{13}$, flexibilidade muscular, motilidade articular, melhora do tônus e força de contração muscular ${ }^{10}$ e principalmente o controle respiratório, já que todos os exercícios são realizados em sincronia com a respiração ${ }^{13}$. Vale ressaltar que existem poucos estudos na literatura relacionados ao isostretching como um todo, e vários pontos de sua atuação efetiva ainda não apresentam uma definição clara quanto aos efeitos terapêuticos. Com isso, o presente estudo tem como objetivo identificar os efeitos do método isostretching no tratamento da escoliose.

\section{Materiais e métodos}

\section{Critérios de elegibilidade}

Foram incluídos na pesquisa os artigos que abordaram a escoliose tratada com a técnica de isostretching, ensaios clínicos randomizados e estudos de casos. Foram excluídos do estudo aqueles artigos que não abordassem a técnica de isostretching como tratamento da escoliose, bem como resumos, tese de doutorado e mestrado, trabalhos de conclusão e estudos que envolveram animais.

\section{Estratégias de Buscas}

Foram pesquisados artigos publicados entre os anos de 1997 a 2017, nas seguintes bases de dados eletrônicos: biblioteca Virtual Scientific Electronic Library Online (SciELO), Google Acadêmico (G.A.), Biblioteca Regional de Medicina (BIREME), Physiotherapy evidence database (PEDro), PubMed e Cochrane. A pesquisa foi realizada em janeiro de 2017 e utilizou os seguintes termos para busca: escoliose (scoliosis), isostretching, isolados e associados em português e em inglês. A busca dos dados foi realizada durante o mês de janeiro de 2017.

\section{Extração de Dados}

Utilizando as palavras-chaves nas bases de dados foram achados 314 artigos [G. A. $(\mathrm{n}=294) ;$ SciELO $(\mathrm{n}=10)$; PEDro $(\mathrm{n}=3)$; PubMed $(n=1)$; Bireme $(n=3)$; Cochrane $(n=3)]$. Após uma leitura minuciosa dos estudos, 306 foram excluídos por não contemplarem os critérios metodológicos. Posteriormente 2 artigos estavam em mais de uma base de dados, restando apenas 6 estudos que contemplavam todos os critérios de inclusão, sendo estes 3 estudos clínicos e 3 rela- 
tos de caso. A Figura 1 mostra o fluxograma da extração dos estudos incluídos na pesquisa.

\section{Classificação de acordo com a escala Physiotherapy Evidence Database (PEDro)}

Somente os três estudos clínicos foram avaliados pela escala PEDro, que é composta por 11 itens, que avaliam a qualidade metodológica de ensaios clínicos, onde os estudos com escore $>5$ são classificados de alta qualidade metodológica e estudos $<5$ de baixa qualidade ${ }^{14}$. Dos ensaios clínicos inclusos, um foi classificado com qualidade metodológica alta e os outros dois com baixa. As pontuações e as descrições dos estudos selecionados estão apresentadas na Tabela 1. Os estudos do tipo relato de caso não puderam ser avaliados pela escala PEDro, haja visto que a mesma não se aplica nestes casos.

\section{Resultados}

Após a análise realizada foram incluídos 6 artigos que contemplaram os critérios metodológicos estipulados. As tabelas foram compostas pelas informações: autor, ano, objetivo, descrição da terapêutica, caracterização da escoliose, resultados.

A Tabela 1 apresenta informações relacionadas aos escores obtidos pelos estudos na escala PEDro. Os pacientes foram submetidos a uma

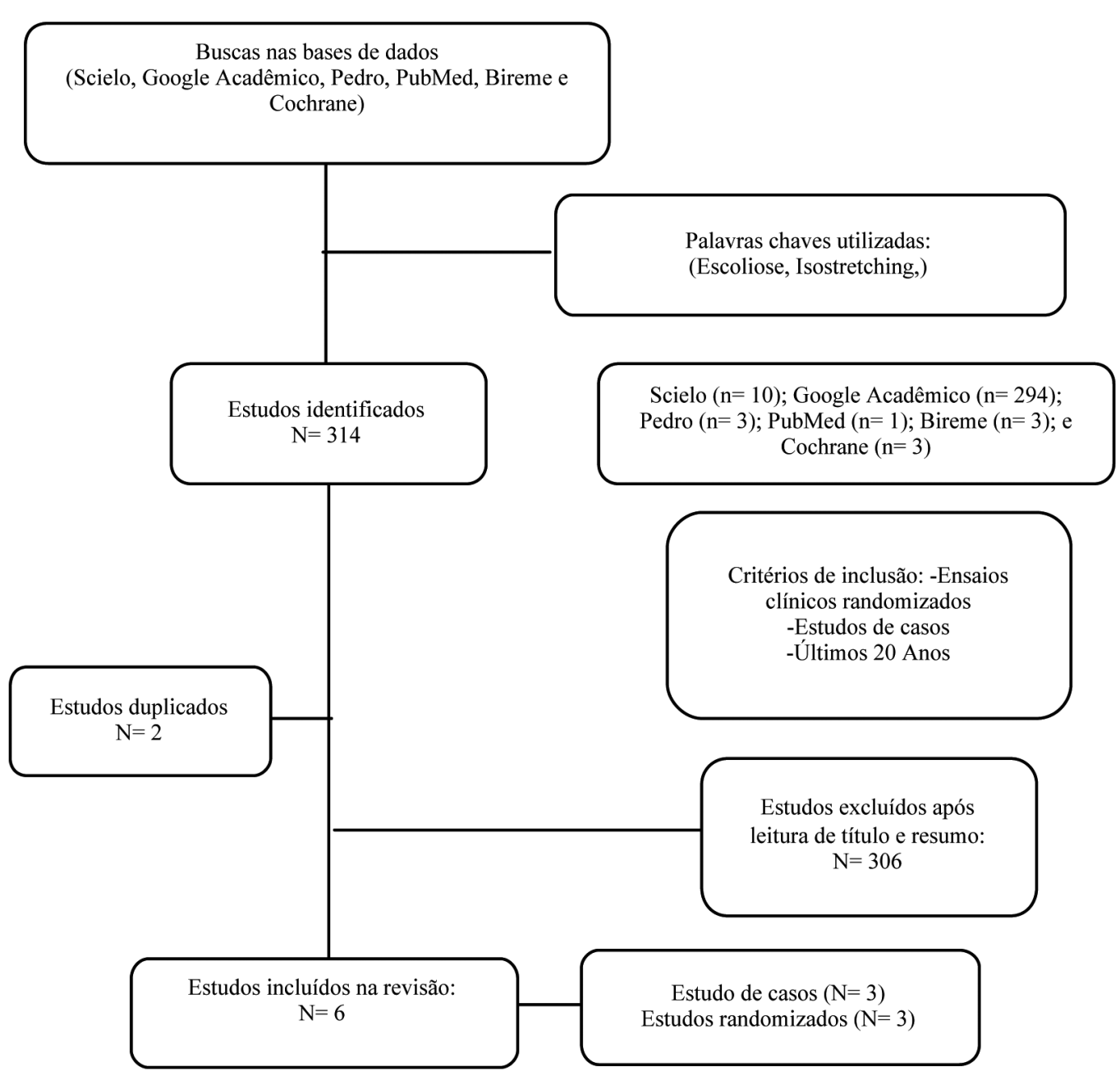

Figura l: Estratégia de busca e seleção dos estudos

Fonte: Autor. 
média de 22 atendimentos com isostreching realizados com duração aproximada de 50 minutos. Após a intervenção os pacientes apresentaram melhoras no alinhamento das curvaturas da co- luna vertebral lombar e torácica, diminuição do ângulo de Cobb e resultados favoráveis em todas as medidas de flexibilidade, dor e qualidade de vida.

Tabela 1: Anólise descritiva dos estudos randomizados com a avaliação da escala PEDro

\begin{tabular}{|c|c|c|c|c|c|}
\hline Autor e ano & Objetivo & Descrição da terapêutica & $\begin{array}{l}\text { Caracterização da } \\
\text { escoliose }\end{array}$ & Resultados & $\begin{array}{c}\text { Escala PEDro } \\
\text { (OM) }\end{array}$ \\
\hline $\begin{array}{c}\text { Monte-Raso e } \\
\text { col., 200915. }\end{array}$ & $\begin{array}{l}\text { O objetivo do } \\
\text { estudo foi avaliar os } \\
\text { efeitos da técnica } \\
\text { isostretching em } \\
\text { alterações posturais. }\end{array}$ & $\begin{array}{l}\text { Os indivíduos foram divididos } \\
\text { em dois grupos segundo o } \\
\text { número de atendimentos, grupo } \\
1 \text { com mais de } 30 \text { atendimentos } \\
\text { e grupo } 2 \text { com menos de } 30 . \\
\text { Atendimentos de } 1 \text { hora, três } \\
\text { vezes por semana. Consistia } \\
\text { em exercícios isométricos } \\
\text { excêntricos, em que o tempo } \\
\text { de manutenção das posturas } \\
\text { é regido por três expirações } \\
\text { profundas e prolongadas, nas } \\
\text { posições deitado, sentado, em } \\
\text { pé, utilizando bola e bastão. }\end{array}$ & $\begin{array}{l}12 \text { voluntários com } \\
\text { aumento da cifose } \\
\text { torácica, escoliose, } \\
\text { protrusão de cabeça e } \\
\text { ombros. }\end{array}$ & $\begin{array}{c}\text { Foi eficaz no } \\
\text { alinhamento da } \\
\text { coluna vertebral } \\
\text { torácica nos dois } \\
\text { grupos, bem como } \\
\text { na melhora da } \\
\text { flexibilidade no } \\
\text { grupo } 1 .\end{array}$ & $\begin{array}{l}5 / 11 \\
\text { Alta }\end{array}$ \\
\hline $\begin{array}{l}\text { Borghi e } \\
\text { col.,2008 }{ }^{16} \text {. }\end{array}$ & $\begin{array}{l}\text { Verificar as } \\
\text { alterações } \\
\text { promovidas } \\
\text { pela prática do } \\
\text { Isostretching sobre } \\
\text { a flexibilidade, a } \\
\text { expansibilidade } \\
\text { torácica e as } \\
\text { curvaturas } \\
\text { escoliótica e lombar } \\
\text { em indivíduos com } \\
\text { escoliose. }\end{array}$ & $\begin{array}{l}\text { Pacientes foram divididos em } \\
\text { três grupos de } 5 \text { pessoas, com } \\
\text { frequência de duas vezes por } \\
\text { semana e duração de } 50 \text { min } \\
\text { por atendimento ou até totalizar } \\
24 \text { atendimentos. Os exercícios } \\
\text { eram realizados nas posições } \\
\text { em pé, sentado e em decúbito } \\
\text { dorsal, com duração proporcional } \\
\text { à expiração forçada, sendo } \\
\text { repetidos por três vezes cada }\end{array}$ & \begin{tabular}{|} 
Selecionados pacientes \\
com idade igual ou inferior \\
a 18 anos. 8 participantes \\
apresentaram escoliose \\
de etiologia idiopática e \\
um, neuromuscular. Com \\
relação ao tipo de curva \\
um possuía escoliose em \\
"S" e o restante escoliose \\
em "C". 8 pacientes \\
apresentaram escoliose \\
tóraco-lombar à esquerda \\
e apenas 1 escoliose \\
torácica à direita.
\end{tabular} & \begin{tabular}{|} 
No final do estudo \\
demonstrou redução \\
do ângulo de Cobb \\
em 5 pacientes \\
e piora em 4 dos \\
casos. Quanto à \\
curvatura lombar, \\
houve média de \\
redução do ângulo \\
de $6,44^{\circ}$.
\end{tabular} & $\begin{array}{l}\text { 4/11 } \\
\text { Baixa }\end{array}$ \\
\hline $\begin{array}{c}\text { Moraes e col., } \\
2011^{17} .\end{array}$ & $\begin{array}{l}\text { O objetivo do } \\
\text { estudo foi verificar } \\
\text { os efeitos do } \\
\text { Isostretching na } \\
\text { dor, flexibilidade, } \\
\text { qualidade de vida } \\
\text { em indivíduos com } \\
\text { escoliose. }\end{array}$ & $\begin{array}{l}\text { O estudo foi realizado com } 15 \\
\text { indivíduos, e os voluntários } \\
\text { foram submetidos a } 12 \text { sessões } \\
\text { de Isostretching, realizadas duas } \\
\text { vezes por semana com duração } \\
\text { de } 50 \text { minutos cada. } 0 \text { método } \\
\text { Isostretching foi aplicado a partir } \\
\text { de posturas pré-determinadas } \\
\text { em decúbito dorsal, sedestação } \\
\text { e bipedestação, realizadas em } \\
\text { três séries, num total de nove } \\
\text { exercícios por sessão, sempre } \\
\text { em associação à contratação dos } \\
\text { principais grupos de músculos } \\
\text { e à expiração prolongada. } \\
\text { Foram utilizados como recursos } \\
\text { terapêuticos tatames, bastões } \\
\text { e bolas }\end{array}$ & \begin{tabular}{|c} 
Selecionados pacientes \\
de ambos os gêneros, com \\
idade entre 18 a 30 anos \\
e que apresentasse como \\
queixa principal alteração \\
postural decorrentes da \\
coluna, com escoliose \\
diagnosticada.
\end{tabular} & $\begin{array}{l}\text { Comparando os } \\
\text { valores obtidos antes } \\
\text { e pós Isostretching } \\
\text { foi possível observar } \\
\text { uma contribuição } \\
\text { favorável em todas } \\
\text { as medidas de } \\
\text { flexibilidade, dor e } \\
\text { qualidade de vida de } \\
\text { forma satisfatória. }\end{array}$ & $\begin{array}{l}\text { 4/11 } \\
\text { Baixa }\end{array}$ \\
\hline
\end{tabular}

Legenda: QM: Qualidade Metodológica.

Fonte: Autor e Escala Physiotherapy Evidence Database (www.pedro.org.au/portuguese/downloads/pedro-scale). 
Na Tabela 2 estão descritos os estudos de casos, nos quais foram utilizados mais pacientes do sexo feminino do que masculino, com idade entre 12 e 19 anos e prevalência de escoliose idiopática. O método foi aplicado com tempo médio de 50 minutos por atendimento. Os resultados obtidos mostraram melhoras significativas no aumento da flexibilidade, padrão postural, e diminuição do ângulo de Cobb, porém no artigo de Sá e col. ${ }^{16}$ não houve modificação na curvatura escoliótica. Em todos os estudos houve uma melhora no quadro álgido e nas retrações das cadeias musculares.

Tabela 2: Anólise descritiva dos relatos de caso incluídos na revisão

\begin{tabular}{|c|c|c|c|c|}
\hline Autor e ano & Objetivo & Descrição da terapêutica & $\begin{array}{c}\text { Caracterização da } \\
\text { escoliose }\end{array}$ & Resultados \\
\hline $\begin{array}{l}\text { Bonorino e } \\
\text { col.,200718. }\end{array}$ & $\begin{array}{c}\text { As principais metas } \\
\text { foram proporcionar } \\
0 \text { alongamento das } \\
\text { cadeias musculares, } \\
\text { aumentar a } \\
\text { flexibilidade e } \\
\text { melhorar o padrão } \\
\text { postural em paciente } \\
\text { com escoliose } \\
\text { através do método } \\
\text { isostreching. }\end{array}$ & $\begin{array}{l}\text { O estudo teve como participante } \\
\text { uma voluntária do sexo feminino } \\
\text { com } 19 \text { anos de idade. } 0 \text { tratamento } \\
\text { foi realizado no período de } 15 \text { de } \\
\text { setembro a } 11 \text { de novembro de } \\
\text { 2007, com três sessões semanais } \\
\text { de uma hora de duração. Durante os } \\
\text { atendimentos foram usadas posturas } \\
\text { extraídas do método Iso-Stretching10 } \\
\text { com o alongamento muscular em } \\
\text { decúbito dorsal, na posição sentada, e } \\
\text { evoluindo para a posição em pé, com } \\
\text { maior grau de dificuldade conforme } \\
\text { a evolução do paciente. Todos os } \\
\text { exercícios foram realizados com uma } \\
\text { respiração profunda e controlada, } \\
\text { visando à mobilidade do diafragma e } \\
\text { do gradil costal. }\end{array}$ & $\begin{array}{c}\text { Diagnóstico de } \\
\text { escoliose tóraco-lombar } \\
\text { dupla a } 2 \text { anos e queixa } \\
\text { principal de dor contínua } \\
\text { e bilateral na região da } \\
\text { curva escoliótica. }\end{array}$ & $\begin{array}{l}\text { Verificou-se melhora do padrão } \\
\text { postural, diminuição da dor, } \\
\text { ganho de flexibilidade, aumento } \\
\text { da amplitude de movimento } \\
\text { e diminuição das retrações } \\
\text { musculares. }\end{array}$ \\
\hline $\begin{array}{c}\text { Sá e } \\
\text { col.,2003 } 200 \text {. }\end{array}$ & $\begin{array}{c}\text { Mostrar os } \\
\text { efeitos do método } \\
\text { Isostretching na } \\
\text { flexibilidade do } \\
\text { paciente portador de } \\
\text { escoliose idiopática. }\end{array}$ & $\begin{array}{l}\text { Indivíduo adolescente, sexo masculino, } \\
13 \text { anos de idade, portador de escoliose } \\
\text { idiopática de grau leve. } 0 \text { tratamento } \\
\text { era composto por duas sessões sema- } \\
\text { nais, com o tempo de } 50 \text { minutos cada, } \\
\text { por um período de } 1 \text { mês, totalizando } \\
11 \text { sessões. } 0 \text { plano de tratamento foi } \\
\text { constituído de posturas sentada, em } \\
\text { pé e deitada. No início do programa } \\
\text { de tratamento, foram escolhidas em } \\
\text { média } 12 \text { posturas. Posteriormente, } \\
\text { a partir da quarta sessão, passaram } \\
\text { a ser realizadas de } 15 \text { a } 20 \text { posturas, } \\
\text { evidenciando assim, maior tolerância ao } \\
\text { alongamento e adaptação às posturas } \\
\text { por parte do sujeito }\end{array}$ & \begin{tabular}{|c|} 
\\
Foi selecionado 1 \\
indivíduo adolescente, \\
sexo masculino, 13 anos \\
de idade, portador de \\
escoliose idiopática de \\
grau leve. A escoliose \\
em questão foi classi- \\
ficada como "escoliose \\
torácica esquerda e \\
tóraco-lombar a direita. \\
\end{tabular} & $\begin{array}{c}\text { Após as } 10 \text { sessões de } \\
\text { fisioterapia realizadas foi possível } \\
\text { comprovar o aumento da flexibi- } \\
\text { lidade geral do paciente, melhora } \\
\text { do alinhamento postural, redução } \\
\text { do quadro álgico, aumento da } \\
\text { ADM dos MMll e coluna vertebral } \\
\text { graças ao desenvolvimento do } \\
\text { alongamento, flexibilidade e } \\
\text { força dos músculos da cadeia } \\
\text { posterior. Não houve alteração } \\
\text { quanto ao ângulo de Cobb das } \\
\text { curvas escolióticas, porém com } \\
\text { o aumento da flexibilidade e } \\
\text { liberação das retrações das } \\
\text { cadeias musculares, houve } \\
\text { diminuição da rotação vertebral }\end{array}$ \\
\hline $\begin{array}{l}\text { Cardoso e } \\
\text { col.,2010 } 20\end{array}$ & $\begin{array}{c}\text { Analisar } \\
\text { alterações clínicas } \\
\text { e radiográficas pré } \\
\text { e pós-tratamento } \\
\text { conservador em } \\
\text { paciente portador de } \\
\text { escoliose idiopática. }\end{array}$ & $\begin{array}{l}0 \text { procedimento fisioterapêutico foi } \\
\text { realizado em três dias da semana, } \\
\text { durante } 50 \text { minutos cada sessão, } \\
\text { totalizando } 30 \text { sessões. As técnicas } \\
\text { de isostreching foram iniciadas na } \\
\text { postura deitada, progredindo para } \\
\text { sentada e evoluindo para em pé. Essa } \\
\text { evolução de posturas foi realizada a } \\
\text { cada dez sessões. }\end{array}$ & $\begin{array}{c}\text { Criança de } 12 \text { anos de } \\
\text { idade, do sexo feminino. } \\
\text { Foi feita a medição } \\
\text { inicialmento do ângulo } \\
\text { de cobb que anterior ao } \\
\text { tratamento era } 42^{\circ} .\end{array}$ & $\begin{array}{l}\text { No final do estudo o paciente } \\
\text { apresentou diminuição da } \\
\text { gibosidade no teste de Adams, } \\
\text { redução do ângulo de Cobb em } 6^{\circ} \\
\text { graus e melhora da postura em } 30 \\
\text { sessões fisioterapêuticas. }\end{array}$ \\
\hline
\end{tabular}

Legenda: ADM: Amplitude de movimento / MMII: Membros inferiores

Fonte: Autor. 


\section{Discussão}

De acordo com os estudos clínicos, o isostretching mostrou-se eficaz em aspectos como a melhora do alinhamento vertebral, redução do ângulo de Cobb, ganho de flexibilidade, aumento da amplitude de movimento, diminuição da dor e benefícios em relação a qualidade de vida dos participantes. Já nos relatos de casos, os resultados encontrados foram o aumento na flexibilidade, melhora no alinhamento postural (com impacto positivo no ângulo de Cobb), ganho de força dos músculos da cadeia posterior, redução álgica e diminuição da gibosidade.

A técnica foi aplicada com exercícios de forma excêntrica sempre em associação com a expiração profunda e realizando a contração dos principais grupos musculares. $\mathrm{O}$ isostretching aplicado com ênfase no fortalecimento dos grupos musculares que atuam na manutenção da postura do corpo, apresenta diminuição das dores lombares, melhora na força muscular ${ }^{21}$. Entretanto o estudo de Pardo e col. (2015) ${ }^{22}$, mensurou a força muscular através de um dinamômetro isocinético e demonstrou que a técnica é pouco eficaz no ganho de força. Uma hipótese levantada pelo autor diz que o isostretching apenas é eficaz em pacientes com disfunções musculoesqueléticas como a escoliose.

Raso et al. (2009) ${ }^{15}$, realizou uma avaliação postural onde os participantes foram fotografados pré e pós tratamento e foram escolhidas 10 posturas do método para serem utilizadas no período de aplicação da técnica. Esse estudo comparou indivíduos que realizaram mais de 30 atendimentos de isostreching com os que realizaram menos de 30. No grupo que teve mais atendimentos, apresentou modificações significativas nas angulações: cifose torácica, triângulo de Talles, e nos ângulos tibiotársico e coxofemoral. Já no grupo com menos atendimentos não obtiveram alterações na avaliação postural significativas.

De acordo com Moraes et al. (2011) ${ }^{17}$, no que diz respeito a flexibilidade, foi possível observar um aumento médio de $15^{\circ}$ para o movimento de flexão lombar, $7,6^{\circ}$ para extensão deste mesmo segmento vertebral, 5, $6^{\circ}$ para inclinação direita e $4,7^{\circ}$ para o movimento de inclinação lombar esquerda após o tratamento com o isostreching. $\mathrm{O}$ que fortalece a ideia que o isostretching pode proporcionar redução da curvatura escoliotica ${ }^{15,16}$.

Em dois estudos de casos também houve diminuição da curvatura da coluna vertebral ${ }^{18,20}$. Sá e col. (2003) ${ }^{19}$, aplicou o método em 11 atendimentos em um adolescente utilizando entre 15 a 20 posturas, e apontou que não houve alteração na mensuração do ângulo de Cobb após a intervenção. Apesar de não ter apresentado alteração no ângulo escoliótico, com o aumento da flexibilidade e diminuição das retrações musculares, houve uma diminuição da rotação da coluna vertebral melhorando o padrão postural do paciente, aumento do alongamento e força dos músculos isquiotibiais e espinhais do tronco, músculos estes que fazem parte da cadeia muscular posterior.

Boroni et al. $(2011)^{18}$, mostraram que na avaliação postural, após a aplicação do isostreching, houve uma diminuição da protrusão dos ombros, melhora no alinhamento da cabeça em relação ao tronco, diminuição da hiperextensão dos joelhos. A avaliação foi realizada de forma subjetiva, não foi possível quantificar os resultados obtidos na pesquisa. O estudo também avaliou a dor que o paciente referia no momento da pesquisa através da escala EVA (escala visual analógica de dor), em que ele apresentou nível 7 ou seja dor moderada. Após ser submetido ao tratamento com a aplicação do isostretching, ele foi avaliado novamente, e apresentou no segundo momento dor nível 2 da escala, melhorando seu quadro álgico após 10 dias de atendimento.

Em outras pesquisas utilizando os métodos e técnicas para o tratamento da escoliose apresentaram resultados parecidos aos do isostretching, como o método Reeducação Postural Global $(\mathrm{RPG}){ }^{\circledR}$, em estudo com 20 indivíduos em que um grupo recebeu a intervenção do RPG® e o grupo controle que não recebeu, os resultados mostraram que o grupo que recebeu o tratamento teve diminuição significativa no ângulo da escoliose, enquanto o grupo controle essa angulação au- 
mentou ${ }^{23}$. Lunes et al. (2010) ${ }^{24}$ utilizou um método antigo para o tratamento de escoliose, mas pouco pesquisado, o Método de Klapp e em comparação com o isostretching, o método utilizou menor número e foi evidenciada diminuição das assimetrias torácicas e modificação da lordose lombar.

As limitações encontradas nesse estudo, foram a carência de pesquisas disponíveis na literatura científica acerca da utilização da técnica isostretching no tratamento da escoliose. $\mathrm{E}$ os poucos estudos disponíveis são relatos de casos, ou seja, são estudos que apresentam pouca qualidade metodológica. Além disso, os estudos randomizados que foram encontrados na literatura, de acordo com a escala PEDro apresentaram uma baixa qualidade. Por isso os efeitos do isostreching não estão esclarecidos e ainda se apresentam desconhecidos clinicamente, porém o presente estudo mostrou resultados favoráveis com a aplicação do método em paciente portadores de escoliose.

\section{Conclusão}

Pode-se concluir que os estudos encontrados sobre a técnica de isostretching na escoliose tiveram resultados positivos, trazendo benefícios no aspecto de dor, diminuição da gibosidade, flexibilidade, força muscular e diminuição da curvatura patológica. Deve-se enfatizar que são necessárias mais pesquisas a respeito do tema dessa revisão e a realização de ensaios clínicos com qualidades metodológicas mais criteriosas devem ser encorajadas.

\section{Referências}

1. Negrini S, Angelo GA, Lorenzo A, Alin BC, Jean $\mathrm{CM}$, Jacek D et al. SOSORT guidelines: orthopaedic and rehabilitation treatment of idiopathic scoliosis during growth. Scoliosis. 2012;7 (3).

2. Konieczny MR, Senyurt H, Krausp R. Epidemiology of adolescent idiopathic scoliosis. J Child Orthop. 2013;7:3-9.
3. Rigo MD, Villagrasa M, Gallo D. A specific scoliosis classification correlating with brace treatment: description and reliability. Scoliosis. 2010;5 (1).

4. Rigo MD, Grivas TB. Rehabilitation schools for scoliosis thematic series: describing the methods and results. Scoliosis. 2010;5 (27).

5. Marques AP. Escoliose tratada com reeducação postural global. Rev. Fisioter. Univ. São Paulo. 1996;3(1/2):65-68.

6. Segura DCA, Nascimento FC, Chiossi CA, Silva MAA, Guilherme JH, Santos JV. Estudo comparativo do tratamento da escoliose idiopática adolescente através dos métodos de rpg. Rev Saúde e Pesq. 2011;4 (2):200-206.

7. Oliveiras AP, Souza de. Tratamento fisioterapêutico em escoliose através das técnicas de isostrecthing e manipulações osteopáticas. Ter Man. 2004;2 (3):104-13.

8. Góis Junior MB. Utilização do Método Klapp no tratamento das escolioses. [trabalho de conclusão de curso]. Alfenas: Universidade José do Rosário Vellano. Curso de Fisioterapia. Departamento de Fisioterapia, 1999.

9. Carvalho AR, Assini TCKA. Aprimoramento da capacidade funcional de idosos submetidos a uma intervenção por isostretching. Rev Bras Fisioter, São Carlos. jul./ago. 2008;12(4):268-73.

10. Junior LCH, Oliveira KTF, Oliveira TGV, Girotto N, Carvalho ACA, Lopes AD. Efeito do método Isostretching na flexibilidade e nível de atividade física em indivíduos sedentários saudáveis. R. bras. Ci. e Mov. 2011;19 (1):26-31.

11. Taborda CF, Moschen GM, Mitsuro MY, Fréz AR, Daniel CR. Método Isostretching como tratamento da dor lombar. R. bras. Qual. Vida, Ponta Grossa. 2014;6 (3):206-215.

12. Silva PHB, Inumaru SMSM. Assessment of pain in patients with chronic low back pain before and after application of the isostreching method. Fisioter. Mov. 2015; 28 (4): 767-777.

13. Macedo CSG, Debiagi PC, Andrade FM. Efeito do isostretching na resistência muscular de abdominais, glúteo máximo e extensores de tronco, incapacidade e dor em pacientes com lombalgia. Fisioter Mov. 2010;23 (1):113-20.

14. The George Institute for Global Health.(21de junho de 1999). Escala de Pedro. Fonte: PEDro phiysiotherapy evidence database: www.pedro.org. au/portuguese/downloads/pedro-scale/ 
15. Raso VVM, Ferreira PA, Carvalho MS, Rodrigues JG, Martins CC, Iunes DH. Efeito da técnica isostretching no equilíbrio postural. Fisioterapia e pesquisa. 2009;16 (2): 137-42.

16. Borghi AF, Antonini GM, Facci LM. Isostretching no tratamento da escoliose: série de casos. Rev Saúde e Pesq. 2008;1(2): 167-171.

17. Moraes JMM, Coelho KO, Carvalho ACA, Prado ERA, Lopes AD. Efeitos do Isostretching na dor, flexibilidade, qualidade de vida e nível de atividade física em adultos com escoliose. Ter Man. 2011; 9 (44):362-365.

18. Bonorino KC, Borin GS, Silva AH. Tratamento para escoliose através do método isostretching e uso de bola suíça. Cinergis. 2007;8 (2): 1-5.

19. Sá AF, Lima IAX. Os Efeitos do Método Isostretching na Flexibilidade do paciente portador de Escoliose Idiopática. Terapia Manual. 2003;2(2):62-68.

20. Cardoso LR, Gonçalves C, Bonvicine C, Barboza MAI. Análise clínica e radiográfica pré e póstratamento conservador na escoliose idiopática do adolescente: estudo de caso. ConScientiae Saúde, 2011;10 (1):166-174.
21. Mann L, Kleinpaul JF, Weber P, Mota CB, Carpes FP. Efeito do treinamento de isostretching sobre a dor lombar crônica: um estudo de casos. Motriz, Rio Claro. 2009; 15 (1):50-60.

22. Pardo MS, Lima AAR, Simões MS, Goya PSA, Voos MC, Caromano FA. Efeito do treino de isostretching na flexibilidade e na força muscular. Acta Fisiatr. 2015;22(2): 72-76.

23. Pasqual MA. Escoliose tratada com reeducação postural global. Fisioter e Pesq. 1996; 3 (1):65-68.

24. Lunes DH, Cecílio MBB, Dozza MA, Almeida PR. Análise quantitativa do tratamento da escoliose idiopática com o método klapp por meio da biofotogrametria computadorizada. Rev bras fisioter. 2010;14(2): 133-40. 